\title{
Variability of Morphological and Agronomical Characteristics of Centella asiatica in Thailand
}

\author{
Namfon Chachai ${ }^{1}$, Banthita Pensuriya ${ }^{1}$, Techita Pinsuntiae ${ }^{1}$, \\ Phattra Pratubkong ${ }^{1}$, Janya Mungngam ${ }^{1}$, Pongsakorn Nitmee ${ }^{1}$, \\ Pongsak Kaewsri ${ }^{1}$, Surasit Wongsatchanan ${ }^{1}$, Rewat Jindajia ${ }^{1}$, \\ Promote Triboun $^{2}$ and Jakkrit Sreesaeng, ${ }^{1, *}$
}

${ }^{1}$ Lamtakong Research Station, Expert Centre of Innovative Agriculture (InnoAg), Thailand Institute of Scientific and Technological Research (TISTR), Pathum Thani 12120, Thailand

${ }^{2}$ National Biobank of Thailand (NBT), National Science and Technology Development Agency (NSTDA), Pathum Thani 12120, Thailand

('Corresponding author's e-mail: Jakkritoneku@gmail.com, Jakkrit@tistr.or.th)

Received: 28 January 2021, Revised: 28 August 2021, Accepted: 1 September 2021

\begin{abstract}
The phenotypic variability of 30 accessions of Asiatic pennywort (Centella asiatica (L.) Urb.) were collected in different regions in Thailand were evaluated. The growth habit among accessions were identified into 2 types including with 20 accessions of erect from and 10 accessions of flatten from. The leaf arrangement divided into 2 types, including with rosette (20 accessions) and spreading leaf (10 accessions). The variability of morphological and agronomical characteristics experiment was derived by Complete Randomized Design (CRD) with 5 replications. The 11 morphological and agronomical character data were collected at 2 mounts after transplant. The phylogenetic tree manifesting the diversity among 30 accessions based on Euclidean distance coefficient identified by 11 morphological and agronomical characteristics. Analysis of variance revealed high and highly significant difference among accessions for all characteristics, shown a wide range of morphological and agronomical characteristics variability of the Asiatic pennywort germplasm. The phylogenetic tree was classified Asiatic pennywort accessions into 2 different clusters with the distance coefficient ranged from $1.00-25.00$. The $1^{\text {st }}$ cluster consisted of 8 accessions and the $2^{\text {nd }}$ cluster consisted of 22 accessions. The $2^{\text {nd }}$ cluster was divided into 2 sub- clusters, including with sub-cluster 2.1 consisted of 21 accessions and sub-cluster 2.2 consisted of 1 accession. Mean value of the $2^{\text {nd }}$ cluster was higher than the $1^{\text {st }}$ group all characters except SPAD Index. The result suggested that could be used accession members in the $2^{\text {nd }}$ cluster can be used for future breeding program of Asiatic pennywort in Thailand.
\end{abstract}

Keywords: Asiatic pennywort, Morphology, Diversity, Correlation, Phenotype

\section{Introduction}

Asiatic pennywort was used as a traditional medicine, food and beverage in India, Malaysia, Thailand and others Southeast Asian countries [1-3]. It has been referred to the ancient traditional Chinese Shennong Herbal about 2,000 years ago and used as Ayurvedic medicine in Indian about 3,000 years ago [4]. The information of traditional medicine revealed that Asiatic pennywort is known to be an antioxidant, anti-inflammatory agent, cognitive enhancer and neuroprotective properties [5]. However, Puttarak et al. [6] reported that the cognitive function improvement by Asiatic pennywort was not strong evidence to support the cognitive effect, its might be improved alertness and relieve anger. While, Orhan [7] reported that Asiatic pennywort could be suggested to be a desired phytopharmaceutical with neuroprotective effect. According with, Hamidpour et al. [8] were found that Asiatic pennywort enhanced memory and cognitive effect.

The research of actives component in Asiatic pennywort were reported in many countries. The actives ingredient of Asiatic pennywort including with saponins, asiaticoside and madecassoside, and their aglycones, asiatic acid and madecassic acids, are the most abundant pentacyclic triterpenoids in all part of Asiatic pennywort [5]. Furthermore, Sudhakaran [9] reported that Asiatic pennywort are richness of several micronutrients, iron, phosphorus, sodium, vitamin $\mathrm{C}$, vitamin A, carotene and dietary fibers. 
Although, several nutrients and active ingredients were founded in all Asiatic pennywort accessions. However, the contents of actives ingredient were depended on accessions and the growth conditions. According with Srivastava et al. [10] reported that the Asiatic pennywort accessions with differed phytogeographical regions in India revealed different concentration of asiaticoside, madeccasoside and asiatic acid. In addition to the biomass and yield components of Asiatic pennywort were different between accessions. Which, Prasad et al. [11] showed that the biomass accumulation of Asiatic pennywort was differed between 4 accessions in India. While, Srithongkul et al. [12] reported that genotypic variations and light intensity were affected on growth characteristics and triterpenoid contents, especially on contents of asiaticoside and madecassoside that the main pharmacologically active triterpenoids of Asiatic pennywort.

The diversity of Asiatic pennywort was differed in many regions of tropical and subtropical area. The morphology and actives ingredient components were depended on the genetic variability and geographical conditions. The studies of Asiatic pennywort diversity were reported in different countries such as, China, Malasia, India, Indonesia, Australia and Thailand [11-16]. The Asiatic pennywort germplasm was differed in the level of phenotypic and genetic variability and differed in the level of international and intranational accessions. Alqahtani et al. [16] reported that different 3 Centella species (C. asiatica, C. cordifolia and C. Erecta) were clustered by using methods based on morphological characters, genetic biodiversity, phytochemical compositions and antioxidant activities. According with, Prasad et al. [11] reported that the variability of Asiatic pennywort germplasm collected from different part of India shown differed biomass accumulation and madecassoside and asiatic acid content. While, Chandrasekara et al. [17] revealed that germplasm evaluation of Asiatic pennywort by using morphological, biochemical and genetic diversity is the essential $1^{\text {st }}$ step in cultivation and breeding efforts. Therefore, the objective of this study aimed to collected and identified the diversity of Asiatic pennywort in Thailand by using morphological and agronomical characteristic data. After that, the collections of Asiatic pennywort could be used to estimate the antioxidants content and used to selected high antioxidant accessions for the future experiments.

\section{Materials and methods}

The 30 accessions of Asiatic pennywort were collected from different locations and habitat in Thailand during October, 2019 - February, 2020. These included with the accessions derived from Northern part (4 accessions), Southern part ( 2 accessions), Northeastern part (17 accessions) and Central part ( 7 accessions), which divided in 2 habitats were cultivated accession (27 accessions) and natural accession (3 accessions). The plant in each accessions were grown under greenhouse condition at Lamthakong Research Station, Nakhon Ratchasima province. The experiment was designed by used Randomized Complete Design (CRD) with 5 replications. The 11 morphological and agronomical character data (plant height, total leaf number, shoot number, leaf per shoot, leaf length, leaf width, petiole length, stolon number, stolon length, panicle number and SPAD index) were collected at 2 mounts after transplant. SPAD index was measured by chlorophyll meter (SPAD 502, Minolta, Japan) with 3 points of fully expanding leaf (tip, middle and base of leaf). Statistical analysis was done by SPSS program version 19. Significant differences $(p<0.05)$ were analyzed by one-way ANOVA followed by Duncan's multiple range tested (DMRT). The correlation coefficient and phenotypic distance based on Euclidean distance coefficient of 30 accessions of Asiatic pennywort were analyzed with 11 morphology and agronomic characteristics data by SPSS program version 19.

\section{Results and discussion}

Locality and habitat of 30 accessions of Asiatic pennywort in Thailand

The diversity of Asiatic pennywort accessions was collected and identified by 11 morphological and agronomical characteristics. The phylogenetic tree manifesting the diversity among 30 accessions of Asiatic pennywort based on Euclidean distance coefficient identified by morphological and agronomical characteristics, analyzed by SPSS program. The location of 30 accessions revealed that Asiatic pennywort were derived from Northern part ( 4 accessions), Southern part ( 2 accessions), Northeastern part (17 accessions) and Central part ( 7 accessions), which divided in 2 habitats were cultivated accession (27 accessions) and natural accession ( 3 accessions). The habitat of 30 accessions were divided into 2 group as cultivated and natural habitat, while plant growth habit revealed that 2 type including with 20 accessions of erect from and 10 accessions of flatten from. The leaf arrangement of Asiatic pennywort revealed that 2 types, including with rosette (20 accessions) and spreading leaf (10 accessions). The 
relative of plant growth habit and leaf arrangement was no related. However, the erect growth habit mostly related to the rosette leaf, while flatten growth habit mostly related to the spreading leaf arrangement (Table 1).

Table 1 The details of Asiatic pennywort accessions were used in this study.

\begin{tabular}{|c|c|c|c|c|c|}
\hline No. & Code & Location & Habitat & Plant growth habit & Leaf arrangement \\
\hline 1 & AP01 & Nakhon Pathom Province & Cultivated & Erect & Rosette \\
\hline 2 & $\mathrm{AP} 02$ & Khon Kaen Province & Cultivated & Erect & Rosette \\
\hline 3 & AP03 & Khunwang District, Chiang Mai Province & Cultivated & Flatten & Spreading \\
\hline 4 & AP04 & Prachinburi Province & Cultivated & Erect & Rosette \\
\hline 5 & AP05 & Phang Nga Province & Along the Beach & Flatten & Spreading \\
\hline 6 & AP06 & Ubon Ratchathani Province & Cultivated & Erect & Rosette \\
\hline 7 & AP07 & Chiang Rai Province & Cultivated & Erect & Rosette \\
\hline 8 & AP08 & Nong Bua Lamphu Province & Cultivated & Flatten & Spreading \\
\hline 9 & AP09 & Chiang Mai Province & Cultivated & Flatten & Spreading \\
\hline 10 & AP10 & Nakhon Phanom Province & Cultivated & Erect & Rosette \\
\hline 11 & AP11 & Nakhon Phanom Province & Cultivated & Erect & Rosette \\
\hline 12 & AP12 & Nakhon Pathom Province & Cultivated & Erect & Rosette \\
\hline 13 & AP13 & Loei Province & Cultivated & Erect & Rosette \\
\hline 14 & AP14 & Yasothon Province & Cultivated & Erect & Rosette \\
\hline 15 & AP15 & Nakhon Ratchasima Province & Cultivated & Erect & Rosette \\
\hline 16 & AP16 & Nakhon Si Thammarat Province & Cultivated & Erect & Rosette \\
\hline 17 & AP17 & Nonthaburi Province & Cultivated & Erect & Rosette \\
\hline 18 & AP18 & Muang District, Nakhon Ratchasima Province & Cultivated & Flatten & Spreading \\
\hline 19 & AP21 & Phra Nakhon Si Ayutthaya Province & Cultivated & Flatten & Spreading \\
\hline 20 & AP22 & Bangkok & Cultivated & Erect & Rosette \\
\hline 21 & AP23 & Yasothon Province & Cultivated & Erect & Spreading \\
\hline 22 & AP24 & Muang District, Nakhon Ratchasima Province & Cultivated & Flatten & Spreading \\
\hline 23 & AP25 & Muang District, Nakhon Ratchasima Province & Cultivated & Flatten & Spreading \\
\hline 24 & AP28 & Chiang Mai Province & Cultivated & Erect & Rosette \\
\hline 25 & AP29 & Loei Province & Weed in Orchard & Flatten & Spreading \\
\hline 26 & AP30 & Wang Nam Khiao District, Nakhon Ratchasima Province & Weed in Orchard & Erect & Rosette \\
\hline 27 & AP31 & Pak Chong District, Nakhon Ratchasima Province & Cultivated & Erect & Rosette \\
\hline 28 & AP32 & Nong Bua Lamphu Province & Cultivated & Flatten & Rosette \\
\hline 29 & AP33 & Pak Chong District, Nakhon Ratchasima Province & Cultivated & Erect & Rosette \\
\hline 30 & AP34 & Nakhon Nayok Province & Cultivated & Erect & Rosette \\
\hline
\end{tabular}

Department for development of Thai Transitional and Alternative Medicine, Ministry of Public Health was announced Asiatic pennywort as Thailand Champion Herbal Products (TCHP) [2]. However, high quality of raw material that used for drug and herbal product are necessary to classified. While, Sakthipriya et al. [18] suggested that a better understanding of genetic diversity and its distribution were essential for its conservation and utilization. Although, the diversity of Asiatic pennywort in Thailand was studied. However, morphology and actives ingredient components were depended on the genetic variability and growing conditions. According with Srithongkul et al. [12] reported that 3 different accessions were collected form Nakon Si Thammarat, Rayong and Ubon Ratchathani province, has been differed morphologies and active ingredients. While, Puttarak and Panichayupakaranant [19] revealed that the raw materials were collected from different part of Thailand has differed pentacyclic triterpenes. Also, different part of plants and harvesting time were affected to the pentacyclic triterpenes as well. This indicated that even genetic and growing conditions cause to the differed active ingredients of Asiatic pennywort.

\section{Cluster analysis and variability of morphology and agronomic characteristic of Asiatic pennywort in Thailand}

The morphological and agronomical characteristic diversity of Asiatic pennywort accessions were investigated under greenhouse condition. The results revealed that 10 morphological and agronomical characteristics were highly significant at $p$-value 0.01 and panicle number was significant at $p$-value 0.05 
(Table 2). The results of plant height shown the highest value in AP02, AP04, AP06, AP07, AP12, AP13, AP17 and AP22 accessions. Total leaf number revealed that AP02 and AP03 were the highest, about 67 and 41.40 leaf per plant, respectively. While, shoot number per plant was the highest in 12 accessions including with AP02, AP03, AP04, AP08, AP09, AP12, AP13, AP20, AP22, AP23, AP24, AP25 and AP28. However, the highest leaf per shoot shown only 4 accessions including with AP06, AP07, AP18 and AP27. This result revealed that leaf length and leaf width of AP02, AP04, AP06, AP07, AP17, AP20, AP23 and AP24 were large lamina than others. While, the petiole length was shown the longest petiole in AP22. AP02 was the most stolon number, the average about 8.60 stolon/plant. Stolon length revealed that AP02, AP03, AP07, AP08, AP12, AP13, AP17, AP22, AP24 and AP25 were the longest stolon. The panicle number of AP13 was the highest about 38.60 panicles. The result of SPAD index shown the lowest leaf greenness unit in AP13 and AP29.

Table 2 Morphological and agronomical characteristic diversity of 30 accessions of Asiatic pennywort in Thailand.

\begin{tabular}{|c|c|c|c|c|c|c|c|c|c|c|c|c|}
\hline No. & Code & $\begin{array}{c}\text { Plant } \\
\text { height } \\
\text { (cm.) }\end{array}$ & $\begin{array}{c}\text { Total leaf } \\
\text { number }\end{array}$ & $\begin{array}{c}\text { Shoot } \\
\text { number }\end{array}$ & $\begin{array}{c}\text { Leaf } \\
\text { per } \\
\text { shoot }\end{array}$ & $\begin{array}{c}\text { Leaf } \\
\text { length } \\
\text { (cm.) }\end{array}$ & 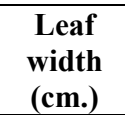 & $\begin{array}{c}\text { Petiole } \\
\text { length } \\
\text { (cm.) }\end{array}$ & $\begin{array}{c}\text { Stolon } \\
\text { number }\end{array}$ & $\begin{array}{c}\text { Stolon } \\
\text { length } \\
\text { (cm.) }\end{array}$ & $\begin{array}{l}\text { Panicle } \\
\text { number }\end{array}$ & $\begin{array}{l}\text { SPAD } \\
\text { index }\end{array}$ \\
\hline 1 & AP01 & $7.30^{\text {cdefghi }}$ & $12.40^{\text {ghij }}$ & $4.40^{\text {efghij }}$ & $2.89^{\mathrm{bcd}}$ & $3.74^{\mathrm{ef}}$ & $2.56^{\text {defgh }}$ & $6.26^{\text {defghi }}$ & $1.40^{\text {fgh }}$ & $20.26^{\text {fghij }}$ & $3.60^{b}$ & $16.80^{\mathrm{abc}}$ \\
\hline 2 & AP02 & $10.80^{\mathrm{ab}}$ & $67.00^{\mathrm{a}}$ & $21.80^{\mathrm{a}}$ & $3.16^{\mathrm{bcd}}$ & $5.94^{\mathrm{ab}}$ & $3.90^{\mathrm{abc}}$ & $8.54^{\text {bcd }}$ & $8.60^{\mathrm{a}}$ & $70.64^{\mathrm{abcd}}$ & $11.60^{\mathrm{b}}$ & $27.60^{\mathrm{a}}$ \\
\hline 3 & AP03 & $7.43^{\text {cdefghi }}$ & $41.40^{\mathrm{ab}}$ & $13.20^{\mathrm{abcd}}$ & $3.00^{\mathrm{bcd}}$ & $3.96^{\mathrm{def}}$ & $2.38^{\text {efgh }}$ & $5.38^{\text {efghij }}$ & $3.40^{\text {bcde }}$ & $43.62^{\text {abcdef }}$ & $6.80^{\mathrm{b}}$ & $17.16^{\mathrm{abc}}$ \\
\hline 4 & AP04 & $10.00^{\mathrm{abc}}$ & $39.60^{\text {bcde }}$ & $13.20^{\text {abcdefg }}$ & $3.06^{\mathrm{bcd}}$ & $4.90^{\mathrm{abcde}}$ & $3.54^{\text {abcde }}$ & $8.50^{\text {bcd }}$ & $3.20^{\text {bcdef }}$ & $42.20^{\text {cdefgh }}$ & $12.80^{\mathrm{b}}$ & $24.90^{\mathrm{ab}}$ \\
\hline 5 & AP05 & $5.00^{\text {hijk }}$ & $5.75^{\mathrm{ij}}$ & $2.75^{\mathrm{ij}}$ & $2.60^{\text {bcde }}$ & $2.10^{\mathrm{gh}}$ & $1.50^{\mathrm{hi}}$ & $4.13^{\text {hijk }}$ & $0.50^{\mathrm{gh}}$ & $7.00^{\mathrm{ij}}$ & $0.25^{\mathrm{b}}$ & $22.90^{\mathrm{ab}}$ \\
\hline 6 & AP06 & $11.50^{\mathrm{ab}}$ & $19.20^{\text {defghij }}$ & $6.00^{\text {defghij }}$ & $3.81^{\mathrm{ab}}$ & $6.46^{\mathrm{a}}$ & $4.10^{\mathrm{ab}}$ & $10.00^{\mathrm{b}}$ & $3.20^{\text {bcdef }}$ & $46.86^{\text {cdefg }}$ & $8.80^{\mathrm{b}}$ & $21.30^{\mathrm{ab}}$ \\
\hline 7 & AP07 & $10.84^{\mathrm{ab}}$ & $37.20^{\text {bcdef }}$ & $10.40^{\text {bcdefghij }}$ & $3.49^{\mathrm{abc}}$ & $5.54^{\mathrm{abcd}}$ & $3.54^{\text {abcde }}$ & $9.30^{\mathrm{bc}}$ & $3.60^{\text {bcdef }}$ & $70.60^{\mathrm{abcd}}$ & $10.00^{b}$ & $18.26^{\mathrm{abc}}$ \\
\hline 8 & AP08 & $6.82^{\text {efghi }}$ & $35.80^{\text {bcdefg }}$ & $15.60^{\mathrm{abcd}}$ & $2.38^{\text {cde }}$ & $3.50^{\mathrm{efg}}$ & $2.40^{\mathrm{efgh}}$ & $5.00^{\text {fghijk }}$ & $3.80^{\text {bcdef }}$ & $65.40^{\mathrm{abcd}}$ & $10.40^{\mathrm{b}}$ & $22.66^{\mathrm{ab}}$ \\
\hline 9 & AP09 & $7.00^{\text {defghi }}$ & $29.20^{\text {bcdefghi }}$ & $12.40^{\text {abcdefgh }}$ & $2.50^{\text {bcde }}$ & $4.20^{\text {cdef }}$ & $2.90^{\text {cdefg }}$ & $4.60^{\text {ghijk }}$ & $4.40^{\mathrm{bc}}$ & $45.00^{\text {cdefg }}$ & $8.20^{\mathrm{b}}$ & $27.40^{\mathrm{a}}$ \\
\hline 10 & AP10 & $7.00^{\text {defghi }}$ & $10.40^{\text {hij }}$ & $3.20^{\text {hij }}$ & $3.15^{\text {bcd }}$ & $4.20^{\text {cdef }}$ & $2.40^{\mathrm{efgh}}$ & $4.60^{\text {ghijk }}$ & $1.40^{\mathrm{fgh}}$ & $14.80^{\text {ghij }}$ & $3.60^{\mathrm{b}}$ & $18.16^{\mathrm{abc}}$ \\
\hline 11 & AP11 & $8.70^{\text {defghi }}$ & $23.20^{\text {cdefghij }}$ & $8.20^{\text {bcdefghij }}$ & $2.87^{\mathrm{bcd}}$ & $4.20^{\text {cdef }}$ & $2.60^{\text {defgh }}$ & $6.10^{\text {defghi }}$ & $2.80^{\text {cdefg }}$ & $45.50^{\text {cdefg }}$ & $9.00^{\mathrm{b}}$ & $19.16^{\mathrm{abc}}$ \\
\hline 12 & AP12 & $9.80^{\mathrm{abcd}}$ & $34.80^{\text {bcdefgh }}$ & $12.60^{\text {abcdefg }}$ & $2.76^{\mathrm{bcd}}$ & $3.50^{\mathrm{efg}}$ & $3.00^{\text {bcdef }}$ & $7.20^{\text {cdefg }}$ & $4.20^{\mathrm{bcd}}$ & $80.60^{\mathrm{a}}$ & $10.80^{\mathrm{b}}$ & $21.50^{\mathrm{ab}}$ \\
\hline 13 & AP13 & $10.20^{\mathrm{abc}}$ & $43.00^{\mathrm{bcd}}$ & $17.20^{\mathrm{abc}}$ & $2.53^{\text {bcde }}$ & $4.20^{\text {cdef }}$ & $2.80^{\text {cdefg }}$ & $7.40^{\text {bcdef }}$ & $5.40^{\mathrm{b}}$ & $79.40^{\mathrm{ab}}$ & $38.60^{\mathrm{a}}$ & $21.60^{\mathrm{ab}}$ \\
\hline 14 & AP14 & $3.56^{\mathrm{jk}}$ & $1.43^{\mathrm{j}}$ & $1.00^{\mathrm{j}}$ & $1.43^{\mathrm{e}}$ & $1.21^{\mathrm{h}}$ & $1.00^{\mathrm{i}}$ & $2.56^{\mathrm{k}}$ & $0.00^{\mathrm{h}}$ & $0.00^{\mathrm{j}}$ & $0.00^{\mathrm{b}}$ & $12.58^{\mathrm{bc}}$ \\
\hline 15 & AP15 & $6.40^{\text {fghij }}$ & $12.60^{\text {fghij }}$ & $5.20^{\text {efghij }}$ & $2.39^{\text {cde }}$ & $3.10^{\mathrm{fg}}$ & $2.10^{\text {fghi }}$ & $4.40^{\text {hijk }}$ & $1.60^{\mathrm{efgh}}$ & $20.20^{\text {fghij }}$ & $4.00^{\mathrm{b}}$ & $19.60^{\mathrm{abc}}$ \\
\hline 16 & AP16 & $7.75^{\text {cdefgh }}$ & $13.75^{\text {fghij }}$ & $6.00^{\text {defghij }}$ & $1.98^{\mathrm{de}}$ & $4.50^{\text {bcdef }}$ & $2.63^{\text {defgh }}$ & $6.75^{\text {cdefgh }}$ & $2.00^{\text {cdefgh }}$ & $38.38^{\text {defghi }}$ & $8.25^{\mathrm{b}}$ & $17.55^{\mathrm{abc}}$ \\
\hline 17 & AP17 & $9.40^{\text {abcde }}$ & $29.00^{\text {bcdefghi }}$ & $11.20^{\text {bcdefgh }}$ & $2.69^{\text {bcde }}$ & $5.60^{\mathrm{abc}}$ & $3.20^{\text {abcdef }}$ & $8.20^{\text {bcde }}$ & $2.60^{\text {cdefg }}$ & $73.60^{\mathrm{abc}}$ & $16.40^{\mathrm{b}}$ & $26.34^{\mathrm{a}}$ \\
\hline 18 & AP18 & $3.80^{\mathrm{jk}}$ & $16.20^{\text {efghi }}$ & $6.20^{\text {defghij }}$ & $3.51^{\mathrm{abc}}$ & $3.10^{\mathrm{fg}}$ & $1.70^{\mathrm{ghi}}$ & $3.60^{\mathrm{ijk}}$ & $1.40^{\mathrm{fgh}}$ & $19.20^{\text {fghij }}$ & $3.80^{\mathrm{b}}$ & $20.02^{\mathrm{abc}}$ \\
\hline 19 & AP21 & $5.00^{\text {hijk }}$ & $18.00^{\text {efghi }}$ & $8.00^{\text {cdefghij }}$ & $2.57^{\text {bcde }}$ & $3.90^{\mathrm{def}}$ & $2.20^{\mathrm{fgh}}$ & $4.70^{\text {fghijk }}$ & $1.80^{\text {defgh }}$ & $31.30^{\text {efghij }}$ & $9.40^{\mathrm{b}}$ & $19.26^{\mathrm{abc}}$ \\
\hline 20 & AP22 & $12.10^{\mathrm{a}}$ & $29.40^{\text {bcdefghi }}$ & $14.00^{\text {abcde }}$ & $2.21^{\mathrm{cde}}$ & $6.00^{\mathrm{ab}}$ & $3.70^{\mathrm{abcd}}$ & $12.80^{\mathrm{a}}$ & $3.20^{\text {bcdef }}$ & $80.80^{\mathrm{a}}$ & $12.80^{\mathrm{b}}$ & $18.06^{\mathrm{abc}}$ \\
\hline 21 & AP23 & $5.10^{\text {hijk }}$ & $25.00^{\text {bcdefghij }}$ & $9.20^{\text {bcdefghij }}$ & $3.17^{\mathrm{bcd}}$ & $3.50^{\text {efg }}$ & $2.10^{\text {fghi }}$ & $5.20^{\text {fghijk }}$ & $2.20^{\text {cdefgh }}$ & $37.50^{\text {defghi }}$ & $9.20^{\mathrm{b}}$ & $23.48^{\mathrm{ab}}$ \\
\hline 22 & AP24 & $4.60^{\mathrm{ijk}}$ & $43.60^{\mathrm{bcd}}$ & $16.00^{\mathrm{abc}}$ & $2.72^{\text {bcde }}$ & $4.20^{\text {cdef }}$ & $2.60^{\mathrm{defgh}}$ & $5.70^{\text {efghij }}$ & $2.40^{\text {cdefgh }}$ & $60.80^{\text {abcde }}$ & $13.80^{\mathrm{b}}$ & $29.04^{\mathrm{a}}$ \\
\hline 23 & AP25 & $7.70^{\text {cdefgh }}$ & $40.20^{\text {bcde }}$ & $13.80^{\text {abcdef }}$ & $3.01^{\mathrm{bcd}}$ & $5.10^{\text {abcde }}$ & $3.90^{\mathrm{abc}}$ & $8.20^{\text {bcde }}$ & $3.60^{\text {bcdef }}$ & $57.30^{\text {abcde }}$ & $12.00^{\mathrm{b}}$ & $23.16^{\mathrm{ab}}$ \\
\hline 24 & AP28 & $9.20^{\text {bcdef }}$ & $35.40^{\text {bcdefg }}$ & $13.60^{\text {abcdef }}$ & $2.95^{\mathrm{bcd}}$ & $6.00^{\mathrm{ab}}$ & $4.30^{\mathrm{a}}$ & $8.40^{\text {bcd }}$ & $3.20^{\text {bcdef }}$ & $51.70^{\text {abcdef }}$ & $15.40^{\mathrm{b}}$ & $25.00^{\mathrm{ab}}$ \\
\hline 25 & AP29 & $3.00^{\mathrm{k}}$ & $13.00^{\text {fghi }}$ & $4.25^{\text {efghij }}$ & $2.57^{\text {bcde }}$ & $2.13^{\mathrm{gh}}$ & $1.45^{\mathrm{hi}}$ & $3.08^{\mathrm{jk}}$ & $1.25^{\mathrm{fgh}}$ & $11.13^{\mathrm{hij}}$ & $4.25^{\mathrm{b}}$ & $15.95^{\mathrm{abc}}$ \\
\hline 26 & AP30 & $4.90^{\text {hijk }}$ & $10.20^{\mathrm{hij}}$ & $4.00^{\text {fghij }}$ & $2.52^{\text {bcde }}$ & $4.30^{\text {cdef }}$ & $2.80^{\text {cdefg }}$ & $6.20^{\text {defghi }}$ & $1.40^{\text {fgh }}$ & $29.90^{\text {efghij }}$ & $6.00^{\mathrm{b}}$ & $21.58^{\mathrm{ab}}$ \\
\hline 27 & AP31 & $6.20^{\text {ghij }}$ & $23.20^{\text {cdefghij }}$ & $5.40^{\text {efghij }}$ & $4.57^{\mathrm{a}}$ & $3.80^{\mathrm{ef}}$ & $2.90^{\text {cdefg }}$ & $5.60^{\text {efghij }}$ & $1.40^{\mathrm{fgh}}$ & $30.20^{\text {efghij }}$ & $4.40^{\mathrm{b}}$ & $24.92^{\mathrm{ab}}$ \\
\hline 28 & AP32 & $5.30^{\text {hijk }}$ & $44.80^{\mathrm{bc}}$ & $17.80^{\mathrm{ab}}$ & $2.97^{\mathrm{bcd}}$ & $4.20^{\text {cdef }}$ & $2.50^{\text {efgh }}$ & $5.60^{\text {efghij }}$ & $2.40^{\text {cdefgh }}$ & $42.90^{\text {cdefgh }}$ & $4.60^{\mathrm{b}}$ & $24.50^{\mathrm{ab}}$ \\
\hline 29 & AP33 & $7.40^{\text {cdefghi }}$ & $7.80^{\mathrm{ij}}$ & $3.60^{\text {ghij }}$ & $2.42^{\text {cde }}$ & $3.80^{\mathrm{ef}}$ & $2.50^{\mathrm{efgh}}$ & $7.30^{\text {cdefg }}$ & $0.60^{\text {gh }}$ & $11.70^{\text {hij }}$ & $2.00^{\mathrm{b}}$ & $7.90^{\mathrm{c}}$ \\
\hline 30 & AP34 & $6.14^{\text {ghij }}$ & $24.60^{\text {bcdefghij }}$ & $9.60^{\text {bcedfghij }}$ & $2.89^{\text {bcd }}$ & $3.70^{\mathrm{ef}}$ & $2.30^{\text {fgh }}$ & $4.00^{\mathrm{ijk}}$ & $1.60^{\mathrm{efgh}}$ & $47.80^{\text {bcdefg }}$ & $9.40^{\mathrm{b}}$ & $22.48^{\mathrm{ab}}$ \\
\hline \multicolumn{2}{|c|}{ F-test } & $* *$ & $* *$ & $* *$ & $* *$ & $* *$ & $* *$ & $* *$ & $* *$ & $* *$ & $*$ & $* *$ \\
\hline \multicolumn{2}{|c|}{$\% \mathrm{CV}$} & 41.1 & 28.7 & 30.2 & 34.0 & 37.0 & 38.9 & 43.5 & 25.4 & 21.9 & 29.4 & 41.6 \\
\hline
\end{tabular}

The result of phenotypic distance based on Euclidean distance coefficient of 30 accessions of Asiatic pennywort were analyzed with 11 morphology and agronomic characteristics data (Table 3). The result revealed that, the clustering carried out on the principal components grouped of the 30 Asiatic pennywort accessions into 2 major clusters (Figure 1). The majority of accessions in cluster 2 was composed of individuals with a high value for the most characteristics. The cluster 2 was divided into 2 sub-clusters, which composed of sub-cluster 2.1 (21 accessions) and sub-cluster 2.2 ( 1 accession). The cluster 1 was composed of accessions with a low value for the most characteristics that including with 8 accessions. The clustering pattern indicated that the existence of a significant amount of variability among 
the Asiatic pennywort collection (Figure 3). The cluster one consisted of the AP01, AP05, AP10, AP14, AP15, AP18, AP29 and AP33 accessions that was characterized by the short plants, less leaves, small leaves, low and short stolon. However, the mean of SPAD index was higher that cluster 2 . The cluster 2 consisted of the AP02, AP03, AP04, AP06, AP07, AP08, AP09, AP11, AP12, AP13, AP16, AP17, AP21, AP22, AP23, AP24, AP25, AP28, AP30, AP31, AP32 and AP34 accessions that was characterized by the taller plant than cluster 1, large leaves, long petiole, more and long stolon and more panicle. Although, the mean of SPAD index was lower than cluster 1 (Table 4).

Table 3 Cluster and number of accessions in each cluster of 30 accessions of Asiatic pennywort.

\begin{tabular}{cccl}
\hline Cluster & Sub-cluster & Number of accession/cluster & \multicolumn{1}{c}{ Accessions } \\
\hline 1 & 1.1 & 8 & AP01, AP05, AP10, AP14, AP15, AP18, AP29, AP33 \\
& & 21 & AP03, AP04, AP06, AP07, AP08, AP09, AP11, AP12, \\
2 & 2.1 & AP13, AP16, AP17, AP21, AP22, AP23, AP24, AP25, \\
& 2.2 & 1 & AP28, AP30, AP31, AP32, AP34 \\
& & &
\end{tabular}

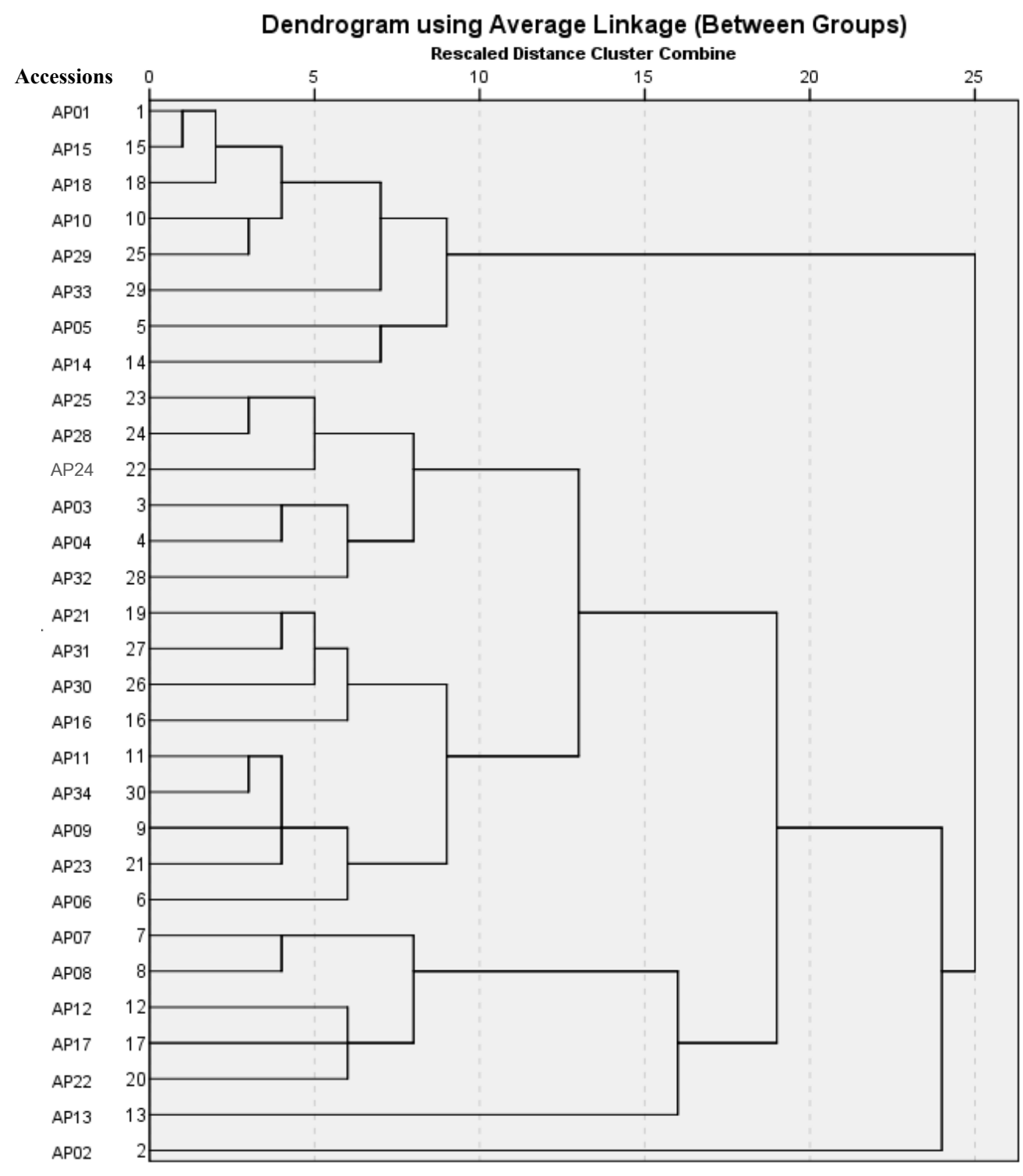

Figure 1 Phylogenetic tree manifesting the diversity among 30 accessions of Asiatic pennywort based on Euclidean distance coefficient identified by morphological and agronomical characteristics. 


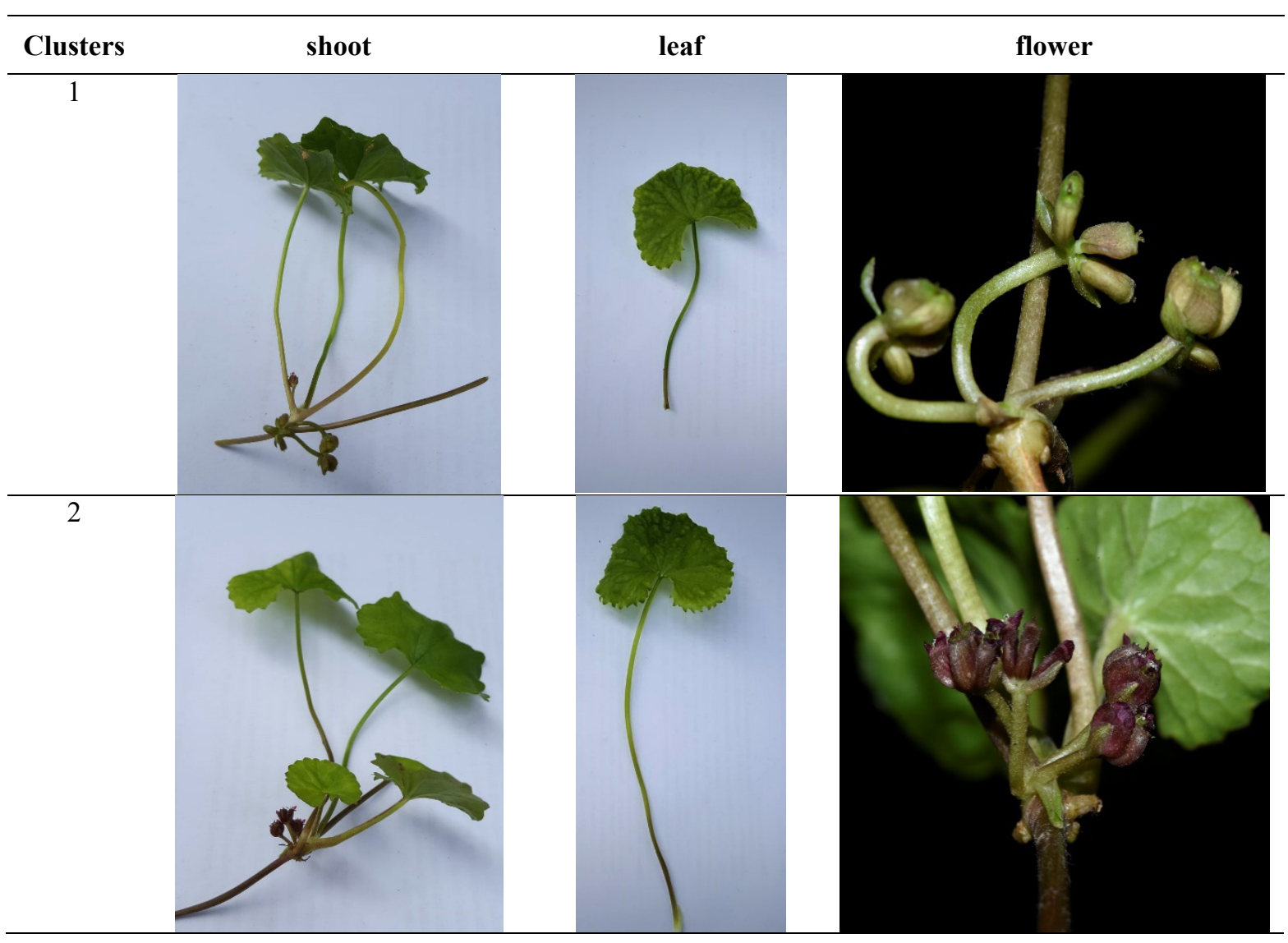

Figure 3 leaf and flower diversity in different 2 clusters of Asiatic pennywort in Thailand.

Table 4 Minimum, maximum, mean value and standard deviation (SD) of 11 agronomic characteristics of the 2 clusters of Asiatic pennywort.

\begin{tabular}{lcccccccc}
\hline \multirow{2}{*}{\multicolumn{1}{c}{ Traits }} & \multicolumn{4}{c}{ Cluster 1 } & \multicolumn{4}{c}{ Cluster 2 } \\
\cline { 2 - 8 } & min & max & mean & SD $^{1 /}$ & min & max & mean & SD $^{1 /}$ \\
\hline 1. plant height (cm.) & 2.00 & 10.50 & 5.43 & 2.40 & 2.50 & 15.00 & 8.01 & 2.92 \\
2. total leaf number & 1.00 & 37.00 & 9.95 & 9.34 & 2.00 & 92.00 & 32.33 & 20.49 \\
3. shoot number & 1.00 & 14.00 & 3.83 & 3.53 & 1.00 & 40.00 & 11.84 & 7.78 \\
4. leaf per shoot & 1.00 & 5.00 & 2.62 & 1.02 & 0.83 & 7.25 & 2.90 & 0.94 \\
5. leaf length (cm.) & 1.00 & 6.20 & 2.92 & 1.26 & 2.00 & 8.50 & 4.61 & 1.38 \\
6. leaf width (cm.) & 0.80 & 3.80 & 1.90 & 0.74 & 1.00 & 6.00 & 3.02 & 1.00 \\
7. Petiole length (cm.) & 1.30 & 9.40 & 4.49 & 2.08 & 2.00 & 16.00 & 6.97 & 2.68 \\
8. stolon number & 0.00 & 4.00 & 1.02 & 1.15 & 0.00 & 13.00 & 3.23 & 2.24 \\
9. stolon length (cm.) & 0.00 & 40.00 & 13.04 & 14.56 & 0.00 & 125.50 & 53.44 & 27.11 \\
10. panicle number & 0.00 & 14.00 & 2.69 & 4.18 & 0.00 & 150.00 & 11.40 & 14.91 \\
11. SPAD index & 0.00 & 49.00 & 16.74 & 10.11 & 2.30 & 39.10 & 22.46 & 7.57 \\
\hline
\end{tabular}

The research of different landraces and accessions variation are important for improvement and plant breeding strategy. Cluster analysis based on morphologies and agronomic characteristics was studied in various plant species, such as pigeon pea, rice and soybean [20-22]. In Asiatic pennywort, Chandrasekara et al. [17] was clustered the 14 morphologies of 5 morphotypes of Asiatic pennywort in Sri Lanka, the results revealed that 2 differed clusters were classified. Ravi et al. [23] identified the variability of 39 accessions of Asiatic pennywort in India based on 14 morphological traits, the results shown wide variability. In the other hand, the classification of Asiatic pennywort based on chromosome and genetic diversity with DNA markers were studied. The results revealed 2 polyploidy complex as diploid $(2 \mathrm{n}=2 \mathrm{x}=18)$ and tetraploid $(2 \mathrm{n}=4 \mathrm{x}=36)$ of Asiatic pennywort in Madagascar [24]. Although, the information of phenotypic diversity of Asiatic pennywort was not closely related to the actives 
ingredients and genetic diversity. However, the results of this study revealed a wide range of variability of morphological characteristics among the 30 accessions of Asiatic pennywort in Thailand.

Correlation coefficient of 11 morphology and agronomic characteristic of Asiatic pennywort

The correlation coefficient of 30 accessions Asiatic pennywort based on 11 morphology and agronomic characteristics were investigated (Table 5). The result revealed that plant height was highly significant positive correlated with petiole length (0.803). Total leaf number was highly significant positive correlated with the total leaf number, stolon number and stolon length $(0.935,0.791$ and 0.710 , respectively). Shoot number was highly significant positive correlated with stolon number and stolon length $(0.758$ and 0.721 , respectively) and negatively correlated with leaf per shoot $(-0.145)$. While, leaf length was highly significant positive correlated with leaf width and petiole length (0.896 and 0.789 , respectively). Leaf width were highly significant positive correlated with petiole length $(0.783)$. The result of stolon number was highly significant positive correlated with stolon length (0.706).

Table 5 Correlation coefficient on morphological and agronomical characteristic diversity of 30 accessions of Asiatic pennywort in Thailand.

\begin{tabular}{|c|c|c|c|c|c|c|c|c|c|c|c|}
\hline Traits & $\begin{array}{c}\text { Plant } \\
\text { height }\end{array}$ & $\begin{array}{c}\text { Total } \\
\text { leaf } \\
\text { number }\end{array}$ & $\begin{array}{c}\text { Shoot } \\
\text { number }\end{array}$ & $\begin{array}{c}\text { Leaf } \\
\text { per } \\
\text { shoot }\end{array}$ & $\begin{array}{c}\text { Leaf } \\
\text { length } \\
\text { (cm.) }\end{array}$ & $\begin{array}{c}\text { Leaf } \\
\text { width } \\
\text { (cm.) }\end{array}$ & $\begin{array}{c}\text { Petiole } \\
\text { length } \\
\text { (cm.) }\end{array}$ & $\begin{array}{c}\text { Stolon } \\
\text { number }\end{array}$ & $\begin{array}{c}\text { Stolon } \\
\text { length } \\
\text { (cm.) }\end{array}$ & $\begin{array}{l}\text { Panicle } \\
\text { number }\end{array}$ & $\begin{array}{l}\text { SPAD } \\
\text { index }\end{array}$ \\
\hline plant height (cm.) & 1 & & & & & & & & & & \\
\hline total leaf number & $0.409^{* *}$ & 1 & & & & & & & & & \\
\hline shoot number & $0.340^{* *}$ & $0.935^{* *}$ & 1 & & & & & & & & \\
\hline leaf per shoot & 0.142 & 0.098 & -0.145 & 1 & & & & & & & \\
\hline leaf length $(\mathrm{cm})$. & $0.657^{* *}$ & $0.459^{* *}$ & $0.415^{* *}$ & $0.220^{* *}$ & 1 & & & & & & \\
\hline leaf width $(\mathrm{cm})$. & $0.657^{* *}$ & $0.449^{* *}$ & $0.387^{* *}$ & $0.212^{* *}$ & $0.896^{* *}$ & 1 & & & & & \\
\hline Petiole length (cm.) & $0.803^{* *}$ & $0.352^{* *}$ & $0.305^{* *}$ & $0.175^{*}$ & $0.786^{* *}$ & $0.783^{* *}$ & 1 & & & & \\
\hline stolon number & $0.461^{* *}$ & $0.791^{* *}$ & $0.758^{* *}$ & 0.026 & $0.493^{* *}$ & $0.500^{* *}$ & $0.389^{* *}$ & 1 & & & \\
\hline stolon length (cm.) & $0.537^{* *}$ & $0.710^{* *}$ & $0.721^{* *}$ & -0.044 & $0.590^{* *}$ & $0.583^{* *}$ & $0.535^{* *}$ & $0.706^{* *}$ & 1 & & \\
\hline panicle number & $0.305^{* *}$ & $0.475^{* *}$ & $0.475^{* *}$ & -0.017 & $0.339^{* *}$ & $0.322^{* *}$ & $0.298^{* *}$ & $0.577^{* *}$ & $0.443^{* *}$ & 1 & \\
\hline SPAD index & $0.195^{*}$ & $0.399^{* *}$ & $0.368^{* *}$ & 0.146 & $0.376^{* *}$ & $0.365^{* *}$ & $0.202^{*}$ & $0.351^{* *}$ & $0.392^{* *}$ & $0.163^{*}$ & 1 \\
\hline
\end{tabular}

** Correlation is significant at the 0.01 level (2-tailed).

* Correlation is significant at the 0.05 level (2-tailed)

The principal component analysis was performed using 11 morphological and agronomical characteristics (Table 6 and Figure 2). The result revealed that only 3 principal components (PCs) exhibited more than 1.0 Eigen values and showed about $86.028 \%$ total variability among the characters were studied. The scree plot used to explain the percentage of variance associated with each principal component. The graph shown the highest variability in PC1 in comparison to the other $10 \mathrm{PCs}$. It indicated that the accessions selected from PC1 might be useful for Asiatic pennywort breeding program.

The results of this study shown that the morphological and agronomical characteristics of Asiatic pennywort closely related more characters. Chomicki et al. [25] reported the Asiatic pennywort stolon was emerged from the oldest leaf axils. While, stolon not only produced new leafy sympodial shoots at each node but also an axillary inflorescence. This indicated that the leafy accession might be produced more stolon, shoot and new leaf faster than accessions that few leaves. While, Aziz et al. [26] reported that Asiatic pennywort leaves contain the highest content of triterpenoids, asiaticoside and madecassoside than root and petiole. Also, the phenotype with smooth leaf has the higher composition of asiaticoside and madecassoside than the fringed leaf. It might be indicated that the morphological characteristic with highly related to leaf traits could be used for selection criteria in Asiatic pennywort breeding program. 
Table 6 Eigen values, variance percentage and cumulative Eigen values percentage of Asiatic pennywort.

\begin{tabular}{lcccc}
\hline \multicolumn{1}{c}{ Traits } & $\begin{array}{c}\text { Principle } \\
\text { components }\end{array}$ & Eigen values & \% of Variance & Cumulative \% \\
\hline Plant height (cm.) & PC 1 & 6.611 & 60.103 & 60.103 \\
Total leaf number & PC 2 & 1.628 & 14.804 & 74.907 \\
Shoot number & PC 3 & 1.223 & 11.122 & 86.028 \\
Leaf per shoot & PC 4 & 0.484 & 4.403 & 90.432 \\
Leaf length (cm.) & PC 5 & 0.430 & 3.910 & 94.342 \\
Leaf width (cm.) & PC 6 & 0.226 & 2.059 & 96.400 \\
Petiole length (cm.) & PC 7 & 0.183 & 1.659 & 98.060 \\
Stolon number & PC 8 & 0.089 & 0.813 & 98.873 \\
Stolon length (cm.) & PC 9 & 0.058 & 0.524 & 99.397 \\
Panicle number & PC 10 & 0.051 & 0.465 & 99.862 \\
SPAD index & PC 11 & 0.015 & 0.138 & 100.000 \\
\hline
\end{tabular}

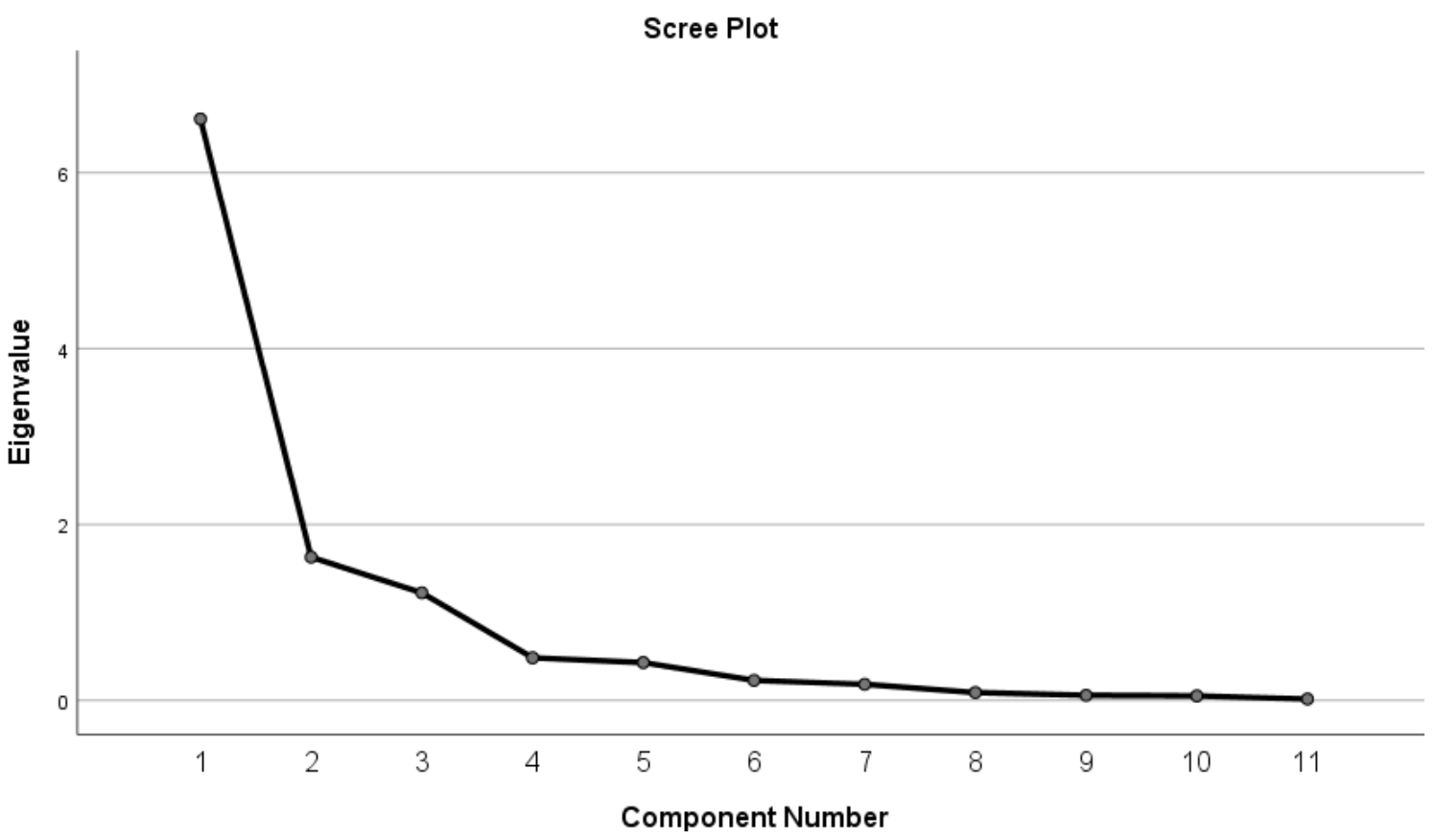

Figure 2 Scree plot of principal component analysis of Asiatic pennywort between eigen value and principal components.

\section{Conclusions}

The variability based on morphological and agronomic characteristics of Asiatic pennywort 30 accessions that collected from various parts of Thailand. The great variability was mainly observed in quantitative characteristics such as, leaf number, leaf length, leaf width, shoot number and stolon number are suitable for Asiatic pennywort germplasm characterization. Cluster analysis based on morphological and agronomic characteristics revealed that the Asiatic pennywort were classified into 2 differed clusters, which cluster 2 shown the highest mean value in the most characters. It indicated that accessions member in cluster 2 growth rapidly and high biomass accumulation. The Asiatic pennywort accessions in cluster 2 could be used for breeding program in the future.

\section{Acknowledgements}

The authors would like to kindly acknowledged the Thailand Institute of Scientific and Technological Research (TISTR) and Thailand Science Research and Innovation (TSRI) for financial and equipment supported. Finally, we would like to thank all staff who involved with this research. 


\section{References}

[1] K Padmalatha and MNV Prasad. Genetic diversity in Centella asiatica (L.) Urb., a memory-enhancing neutraceutical herb, using RAPD markers. Med. Aromat. Plant Sci. Biotechnol. 2008; 2, 90-5.

[2] P Yasurin, M Sriariyanun and T Phusantisampan. Review: The bioavailability activity of Centella asiatica. KMUTNB Int. J. Appl. Sci. Tech. 2016; 9, 1-9.

[3] P Hashim. Centella asiatica in food and beverage applications and its potential antioxidant and neuroprotective effect. Int. Food Res. J. 2011; 18, 1215-22.

[4] European Medicines Agency. Assessment report on Centella asiatica (L.) Urban, herba. United Kingdom, 2010, p. 1-44.

[5] NE Gray, AA Magana, P Lak, KM Wright, J Quinn, JF Stevens, CS Maier and A Soumyanath. Centella asiatica - phytochemistry and mechanisms of neuroprotection and cognitive enhancement. Phytochem Rev. 2018; 17, 161-94

[6] P Puttarak, P Dilokthornsakul, S Saokaew, T Dhippayom, C Kongkaew, R Sruamsiri, A Chuthaputti and $\mathrm{N}$ Chaiyakunapruk. Effects of Centella asiatica (L.) urb. on cognitive function and mood related outcomes: A systematic review and meta-analysis. Sci. Rep. 2017; 7, 10646.

[7] IE Orhan. Centella asiatica (L.) urban: From traditional medicine to modern medicine with neuroprotective potential. Evid.based Complement. Altern. Med. 2012; 2012, 946259.

[8] R Hamidpour, S Hamidpour, M Hamidpour, M Zarabi, M Sohraby and R Hamidpour. Medicinal property of Gotu kola (Centella asiatica) from the selection of traditional applications to the novel phytotherapy. Arch. Cancer Res. 2015; 3, 1-7.

[9] MV Sudhakaran. Botanical pharmacognosy of Centella asiatica (Linn.) urban. Pharmacogn. J. 2017; 9, 546-58.

[10] S Srivastava, S Tiwari, N Srivastava, S Verma and AKS Rawat. Chemotaxonomic studies on Centella asiatica (L.) urb. from varied phytogeographical conditions of India for its industrial prospection. Proc. Natl. Acad. Sci., India, Sect. B Biol. Sci. 2019; 89, 1057-66.

[11] A Prasad, SS Dhawan, AK Mathur, O Prakash, MM Gupta, RK Verma, RK Lal and A Mathur. Morphological, chemical and molecular characterization of Centella asiatica germplasms for commercial cultivation in the Indo-Gangetic plains. Nat. Prod. Commun. 2014; 9, 779-84.

[12] J Srithongkul, S Kanlayanarat, V Srilaong, A Uthairatanakij and P Chalermglin. Effects of light intensity on growth and accumulation of triterpenoids in three accessions of Asiatic pennywort (Centella asiatica (L.) urb.). J. Food Agric. Environ. 2011; 9, 360-3.

[13] XG Zhang, T Han, ZG He, QY Zhang, L Zhang, K Rahman and LP Qin. Genetic diversity of Centella asiatica in China analyzed by inter-simple sequence repeat (ISSR) markers: Combination analysis with chemical diversity. J. Nat. Med. 2012; 66, 241-7.

[14] BW Gbolahan, AI Abiola, J Kamaldin, MA Ahmad and MS Atanassova. Accession in Centella asiatica; current understanding and future knowledge. J. Pure Appl. Microbio. 2016; 10, $2485-94$.

[15] HM Mumtazah, Supriyono, Y Widyastuti and A Yunus. The diversity of leaves and asiaticoside content on three accessions of Centella asiatica with the addition of chicken manure fertilizer. Biodiversitas. 2020; 21, 1035-40.

[16] A Alqahtani, JL Cho, KH Wong, KM Li, V Razmovski-Naumovski and GQ Li. Differentiation of three Centella species in Australia as inferred from morphological characteristics, ISSR molecular fingerprinting and phytochemical composition. Front. Plant Sci. 2017; 8, 1980.

[17] CHWMRB Chandrasekara, RAPI Sumanarathne and PCG Bandaranayake. Centellaasiatica morphotypes differ genetically as well as macronutrients content, total phenolic content and chemical fingerprints of leaves. J. Agric. Sci. Sri Lanka. 2020; 15, 75.

[18] M Sakthipriya, SS Vishnu, S Sujith, PR Kumar and KK Sabu. Analysis of genetic diversity of Centella asiatica using SSR markers. Int. J. Appl. Sci. Biotechnol. 2018; 6, 103-9.

[19] P Puttarak and P Panichayupakaranant. Factors affecting the content of pentacyclic triterpenes in Centella asiatica raw materials. Pharm. Biol. 2012; 50, 1508-12.

[20] F Zavinon, H Adoukonou-Sagbadja, A Bossikponnon, H Dossa and C Ahanhanzo. Phenotypic diversity for agro-morphological traits in pigeon pea landraces (Cajanus cajan L.) millsp. cultivated in southern Benin. Open Agric. 2019; 4, 487-99.

[21] NL Hien, WA Sarhadi, Y Oikawa and Y Hirata. Genetic diversity of morphological responses and the relationships among Asia aromatic rice (Oryza sativa L.) cultivars. Tropics 2007; 16, 343-55.

[22] S Kachare, S Tiwari, N Tripathi and VV Thakur. Assessment of genetic diversity of soybean (Glycine $\max$ ) genotypes using qualitative traits and microsatellite markers. Agr. Res. 2020; 9, 2334. 
[23] CS Ravi1, K Umesha, K HimaBindu, GR Shetty and GSA Kumar. Collection and morphological variability in ecotypes of Indian pennywort (Centella asiatica L.) of hill zone of Karnataka, India. Int. J. Curr. Microbiol. App. Sci. 2019; 8, 994-1008.

[24] SOR Rakotondralambo, M Rodier-Goud, R Rivallan, A Lussert, P Danthu, F Lamotte, E Ralambofetra, P Ramavovololona, JL Noyer and FC Baurens. Insight into the biology, genetics and evolution of the Centella asiatica polyploidy complex in Madagascar. Ind. Crop Prod. 2013; 47, 118 25.

[25] G Chomicki, YM Staedler, LPR Bidel, C Jay-Allemand, J Schönenberger and SS Renner. Deciphering the complex architecture of an herb using micro-computed X-ray tomography, with an illustrated discussion on architectural diversity of herbs. Bot. J. Linn. Soc. 2018; 186, 145-57.

[26] ZA Aziz, MR Davey, JB Power, P Anthony, RM Smith and KC Lowe. Production of asiaticoside and madecassoside in Centella asiatica in vitro and in vivo. Biol. Plant. 2007; 51, 34-42. 